\title{
First report of stem canker of Rosa spp. caused by Didymella pomorum in Canada
}

\author{
Evgeny llyukhin ${ }^{1}$ (D
}

Received: 19 June 2021 / Accepted: 22 November 2021 / Published online: 20 January 2022

(c) Società Italiana di Patologia Vegetale (S.I.Pa.V.) 2021

Keywords Ontario $\cdot$ Stem Canker $\cdot$ Canada $\cdot$ Rosa

Rosa is an important genus in the Rosaceae family and includes over 200 species (Gudin 2000). In Summer 2020, stem cankers were observed on rose plants in Southern Ontario, Canada. Up to $60 \%$ of plants were found to be cankered. Symptomatic plants were collected, sectioned, surface sterilized, rinsed with sterile water, and dried. Stem segments were then plated on malt extract agar (MEA) and incubated at room temperature in the dark. After 4 days, hyphal tips were transferred to fresh MEA plates and incubated as described. The fungal colonies that emerged from rose samples were fast-growing, grey-brown, forming aerial mycelium. Conidia were abundant, ellipsoid and dark brown $(3.6 \times 2.2 \mu \mathrm{m}, \mathrm{N}=50)$. Sequence analysis of the internal transcribed spacers (ITS) and of the large subunit rRNA (LSU) was performed and the obtained ITS (MZ379419, MZ379420) and LSU (MZ928446, MZ928447) sequences both had $100 \%$ identity with the Coniothyrium pyrinum strain (MH856053, MH867548 (CBS 110.40)). The sequences obtained from our samples clustered with $C$. pyrinum strains isolated from Glaucium flavum and formed a distinct clade with strong bootstrap support based on the maximum parsimony and the maximum likelihood analyses. C. pyrinum is currently a synonym of Didymella pomorum (Chen et al. 2015). For pathogenicity tests, ten healthy rose plants were inoculated with the fungus mycelium placed on a wound generated with a scalpel. Three control plants were inoculated as above but using a sterile medium. The plants were kept outdoors with normal temperature and humidity. After fourteen days, the necrotic lesions were clearly observed. No disease symptoms were noticed for control plants. The fungus was re-isolated from eight of ten inoculated plants. The species has been reported to be associated with branch dieback of almond trees in India (Fazli and Razdan 1991), but, based on available information, this is the first record of $D$. pomorum in Canada on this host.

\section{Declarations}

Conflict of interest The authors have no conflicts of interest to declare that are relevant to the content of this article.

\section{References}

Chen Q, Jiang JR, Zhang GZ, Cai L, Crous PW (2015) Resolving the Phoma enigma. Stud Mycol 82:137-217

Fazli MA, Razdan VK (1991) A new record of Coniothyrium pyrinum on almond trees. Indian Phytopathol 44(3):422

Gudin S (2000) Rose: genetics and breeding. Plant Breed Rev 17:159-189

Publisher's Note Springer Nature remains neutral with regard to jurisdictional claims in published maps and institutional affiliations.

Evgeny Ilyukhin

evgeny.ilyukhin@gmail.com; eilyukhin@brocku.ca

1 Department of Biological Sciences, Brock University,

St. Catharines, ON, Canada 